\title{
Benchmarking the performance of density functional theory based Green's function formalism utilizing different self-energy models in calculating electronic transmission through molecular systems
}

\author{
Alexander Prociuk, Ben Van Kuiken, and Barry D. Dunietz ${ }^{\mathrm{a})}$ \\ Department of Chemistry, University of Michigan, Ann Arbor, Michigan 48109
}

(Received 29 June 2006; accepted 19 October 2006; published online 30 November 2006)

\begin{abstract}
Electronic transmission through a metal-molecule-metal system is calculated by employing a Green's function formalism in the scattering based scheme. Self-energy models representing the bulk and the potential bias are used to describe electron transport through the molecular system. Different self-energies can be defined by varying the partition between device and bulk regions of the metal-molecule-metal model system. In addition, the self-energies are calculated with different representations of the bulk through its Green's function. In this work, the dependence of the calculated transmission on varying the self-energy subspaces is benchmarked. The calculated transmission is monitored with respect to the different choices defining the self-energy model. In this report, we focus on one-dimensional model systems with electronic structures calculated at the density functional level of theory. (C) 2006 American Institute of Physics. [DOI: 10.1063/1.2397676]
\end{abstract}

\section{INTRODUCTION}

Molecular electronics is a field with increasing interest and recent developments. ${ }^{1-17}$ In this field, the building block is a molecular electronic transport junction, which consists of one to few molecules immersed between two conducting electrodes. This molecular structure responds to an applied potential bias across the electrodes by allowing electron current to pass through. The merit of this field stems from the prospect of fabricating highly efficient electronic devices harvesting the unique properties of single molecules. ${ }^{2,11,12,18}$ This may offer the ultimate miniaturization and accuracy limit. The success of experiments in measuring charge transfer through molecular wires (MWs) has also emphasized the need for reliable theoretical modeling.

Computational modeling plays an important role in the development of molecular electronics research. Computational studies provide significant insight into the electron transport process. Different theoretical schemes have been employed to model MW conductivity. Most are based on viewing the conductivity as scattering events through the molecule which connects two electronic baths. ${ }^{19-21}$ In this description, conductance is related to the ability of the carriers to pass through the sample. Transport is therefore determined by the scattering properties of the occupied electronic states involved in the process under the influence of the external potential bias. This coherent process can be described by concentrating on the molecular scattering states and the probability of these states to transmit electrons through the sample. This view is often referred to as the Landauer scheme for describing quantum transport, which is widely used for molecular conductance modeling. $16,18,22-26$

The first two ab initio descriptions of MWs, implementing the Landauer view of conductance, employed

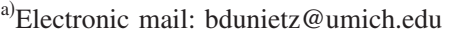

Hartree-Fock ${ }^{23}$ and density functional theory ${ }^{24}$ (DFT) levels. Additional recent $a b$ initio studies employed DFT to accumulate information on the nature of the interaction between the MW and the leads underlying the electron transport (ET) process. ${ }^{27-29}$ More recent advances implement a refined version where dynamical effects due to the conductance are incorporated by letting the electronic density respond selfconsistently to the electron baths and the applied potential bias. ${ }^{25,28,30-35}$ This nonequilibrium description is a direct extension of the static Green's function (GF) formalism. The nonequilibrium GF (NEGF) scheme addresses a major caveat in basic GF formalism, where the limitation of using an elastic view of conductance is lifted. However, NEGF requires an expansive solution for self-consistency of the density and the response to the potential bias. In this report we focus on the convergence of the treatment of the moleculesurface interaction for molecular conductance modeling within the Landauer picture based on full self-consistent field (SCF) electronic structure calculations.

The treatment of the molecule-surface interaction, where the effect of the bulk on the molecular electronic states has to be well described, is a fundamental challenge in modeling molecular conductance. The coupling of a molecule to the macroscopic electrodes mixes the discrete molecular levels with the continuum of states in the metal. New molecular states emerge from the high densities of the metal. Furthermore, conduction is an irreversible process that can be defined only with the presence of the dissipating bath. Thus, conductivity calculations need to address the interaction between the molecule and its environment in nonequilibrium conditions.

The use of a truncated model in describing conductivity leads to clear difficulties arising from the varying number of electrons (due to conductivity) within the model. This has been addressed by different approaches. The simplest involves the jellium models for representing the leads. ${ }^{24,27,36,37}$ 
More elaborate approaches use self-energy (SE) models to represent the coupling of the molecule to the lead. ${ }^{38}$ The SE matrices are projections of the bulk electronic properties of the electrode, which is described by its chemical potential, to a finite subspace under the influence of the adsorbed molecule. The quality and convergence of the self- energy models is expected to have similar trends at two levels of DFT based theories. ${ }^{39}$ These levels include the more basic GF formalism implementing the Landauer description and the higher level implementation of NEGF methodology involving the response of the electronic density to the bias.

The SE models are defined by providing limits on the device region and the bulk repeating unit. In principle, this partitioning should not affect integrated calculated properties, such as conductivity, when the models used in the calculation are well converged. However, if, for example, only a margin of the surface, which is greatly altered by the adsorption, is included in the calculation, the model is bound to fail in describing faithfully transport properties.

Clearly, the ability to provide a reliable description where the effect due to model partitioning becomes negligible depends on the inclusion of enough atoms of the electrodes in the device region. It is well known, for example, that thiol groups bond covalently to gold atoms. Therefore, simple models where such gold atoms are included in the electrode region (instead of the device region) are expected to lead to substantial errors. This has been demonstrated recently by Solomon et al. In their study large gold clusters had to be included within the device to obtain converged conductance descriptions. ${ }^{40}$ Note that their electronic structure calculations were performed at the semiempirical level.

Here we describe a rigorous study on the adequacy of a widely used scheme for representing the bulk in conductivity modeling. In this scheme the bulk is represented through partitioning the model system implementing self-energies as outlined below. ${ }^{25,28,32,34,41,42}$ The reliability of the approximations inherent in the model can be verified by varying the partitioning for the same calculated electronic density. The required conditions (defining the SEs), where arbitrariness in the transport description due to these choices is eliminated, are determined. This determines the adequacy of SEs defined for a variety of systems. In this study, various choices of SE subspaces are used to simulate a simple MW extended between two bulk wires of gold or other metal atoms. In the next section, we review the GF based formalism for calculating transmission. We then describe the molecular model system used for benchmarking the convergence of the SE models. The analysis of the convergence follows by focusing on different factors crucial to the modeling. These factors include the GF used to describe the bulk, the limit of the device, the extent of electronic delocalization within the bulk, and the size of the cluster model. This knowledge is needed to implement converged and reliable transmission calculations.

\section{GREEN'S FUNCTION FORMALISM FOR ELECTRONIC TRANSMISSION}

Most molecular conductivity calculations are based on the Landauer description where molecular conductance is as- sociated with noninteracting scattering electrons. ${ }^{21}$ In this approach conductivity is evaluated by integration over quantum mechanical transmission,

$$
I(V)=e / h \int T(E, V)\left[f_{l}\left(E, \mu_{l}\right)-f_{r}\left(E, \mu_{r}\right)\right] d E,
$$

where $T$ is the (molecular) transmittance function and $f_{l}$ and $f_{r}$ are the Fermi distributions of the leads. The transmission function peaks at different molecular electronic levels due to scattering events through the conductor.

In calculating the transmission, the infinite system is represented by an extended molecular model. In this picture the system is subdivided into three parts, i.e., the left and right electrodes and the molecular scattering region. $8,18,22-24,26$ The (semi-infinite) electrodes are represented by Green's functions calculated for these left and right sections. The effect of the infinite bulk on the molecule is described by projecting the bulk GFs $\left(g_{l}\right.$ and $\left.g_{r}\right)$ on the central molecule using electronic coupling terms to obtain the bulk self-energies $(\Sigma)$ :

$$
\Sigma_{l}(E)=\left(E S_{c l}-H_{c l}\right) g_{l}\left(E S_{l c}-H_{l c}\right)
$$

where $H_{c l}$ and $S_{c l}$ are the Hamiltonian and overlap electronic bulk-device coupling integral matrices. The GF of the central molecule is then calculated with the effect of the semiinfinite bulk represented by the SE matrix:

$$
G_{c}=\left(E S-H_{c c}-\left(\Sigma_{l}+\Sigma_{r}\right)\right)^{-1} .
$$

The bulk's GF $\left(g_{l}\right)$ represents the electronic structure of the semi-infinite electrode and can be calculated with a tightbinding (TB) scheme:

$$
g_{l}=\left(\alpha-\beta g_{l} \beta^{T}\right)^{-1},
$$

where the electronic coupling terms between neighboring units are described by $\beta$ and the diagonal sub-blocks are represented by $\alpha$.

The TB solution for the surface GF [Eq. (4)] is accelerated by solving for the surface and bulk GFs simultaneously. ${ }^{43,44}$ In this implementation, recursion relations between the electronic coupling and the bulk's GF are used to efficiently treat the effect of an infinite number of bulk layers:

$$
\begin{aligned}
\gamma_{i+1}(E)= & \gamma_{i}(E) g_{b, i}(E) \gamma_{i}(E), \\
\beta_{i+1}(E)= & \beta_{i}(E) g_{b, i}(E) \beta_{i}(E), \\
g_{s, i+1}(E)= & {\left[g_{s, i}^{-1}(E)-\gamma_{i}(E) g_{b, i}(E) \beta_{i}(E)\right]^{-1}, } \\
g_{b, i+1}(E)= & {\left[g_{b, i}^{-1}(E)-\gamma_{i}(E) g_{b, i}(E) \beta_{i}(E)\right.} \\
& \left.-\beta_{i}(E) g_{b, i}(E) \gamma_{i}(E)\right]^{-1},
\end{aligned}
$$

where $g_{s}$ is the surface GF, $g_{b}$ is the bulk GF, and the process is initiated by the electronic integral matrices calculated at the appropriate DFT level with

$$
g_{b, 0} \equiv g_{s, 0}=\alpha^{-1}, \quad \gamma_{i, 0}=\beta^{\dagger}, \quad \beta_{i, 0}=\beta .
$$

This calculation is repeated at every energy in the integration grid. Alternatively, the effect of the metal can be assumed to be constant in energy. This constitutes the widely used wide 

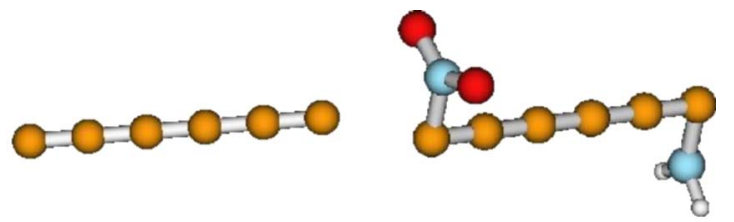

$\mathrm{C}_{6}$

(a)

(b)

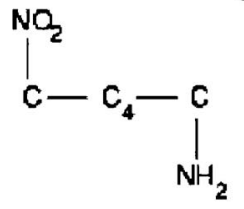

FIG. 1. The molecular systems considered include a cumulene bonded between gold or aluminum wires. In addition we have considered a functionalized cumulene as indicated by the $\mathrm{NO}_{2}$ and $\mathrm{NH}_{2}$ polarizing groups added to the edge carbons. We have also considered bent structures where the cumulene is not on the line defined by the two electrodes.

band limit (WBL). An additional approximation is to assume that the metal is dominated by a single band, which results in a constant GF of the bulk. This may be implemented, for example, for gold electrodes, where the density of states (DOS) is dominated by the $S$ band. ${ }^{18}$ These two levels of bulk representations are employed and the effects of using the corresponding SEs are compared and benchmarked below.

The quantum transmission is then evaluated from the device Green's $\left(G_{c}\right)$ and broadening functions $\left(\Gamma_{l, r}\right){ }^{45}$

$$
T(E, V)=\operatorname{tr}\left[G_{c}^{R} \Gamma_{l} G_{c}^{A} \Gamma_{r}\right] .
$$

The broadening functions describe the effect of the random scattering events in the metal contacts on the molecular states. These functions are related to the coupling of the conductor to the contacts (the self-energies) by

$$
\Gamma=i\left[\Sigma-\Sigma^{\dagger}\right] \text {. }
$$

\section{MODELS}

The benchmarking is performed on a set of onedimensional model systems. The molecular device is based on a linear chain of carbon atoms (cumulene) and is depicted in Fig. 1. A chemically more complex system, where a pair of electron withdrawing and donating groups is added, is also considered. This is achieved by bonding a $\mathrm{NO}_{2}$ to one edge carbon and a $\mathrm{NH}_{2}$ group to the other edge carbon. This allows us to generalize the considered electronic structure features that are important for electron transport properties. These cumulenes are then bonded to one-dimensional electrodes, as depicted in Fig. 2.

Conductance studies employing atomistic gold have been applied, for example, to study magnetoresistance of molecular devices. ${ }^{46-48}$ One-dimensional atomic bulk models have been shown to reproduce dominant trends observed with larger surface models employing the Landauer description as well. ${ }^{49}$ In addition, this is an important model system simulating a single atom link between the thiol and the gold surface which exists in several experimental setups as indicated by Tao and co-workers. ${ }^{50,51}$ In their experiments, a scanning tunneling microscope (STM) tip is used to pull a string of gold atoms off the surface, ${ }^{52,53}$ leading eventually to

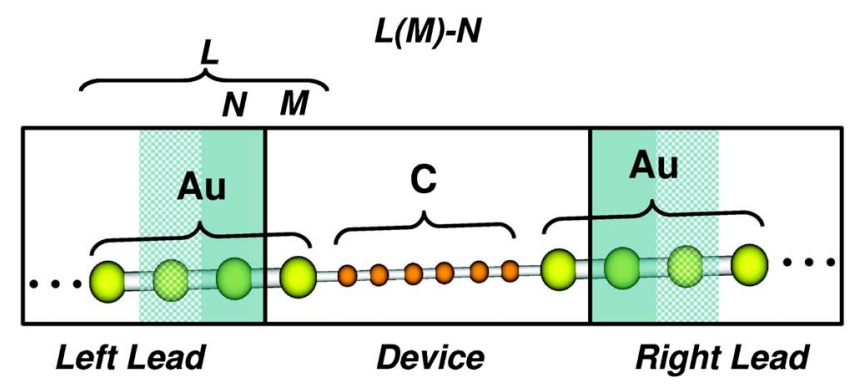

FIG. 2. The self-energy models are defined by three integer values as indicated in the figure. $L$ denotes the total number of metal atoms included in the cluster calculation for representing each electrode. $M$ is the number of metal layers included with the device after partitioning the system and $N$ defines the length of interatom interactions included in defining the bulk. The subspace defined by $N$ is the repeating unit assumed for the periodic bulk material.

the ability to bind a single dithiolated molecule between two fragments of a gold atom wire. In addition we also consider variants of these systems where the bulk consists of $\mathrm{Al}$ atoms and/or the molecular geometry is allowed to bend out of the line defined by the electrodes. Transmission calculations implement the Green's function formalism as described above on these different systems, where the transport is considered to occur through the molecular device immersed between the two semi-infinite bulk materials. Below we focus on results obtained with the gold model. The other considered systems feature similar trends.

Within the GF formalism the effect of the infinite bulk on the molecular system is achieved by projection on predefined subspaces. This involves calculating the bulk's self energy models. Therefore, the implementation of the Green's function formalism requires several choices. These define the size of the immersed device, namely, the number of metal atoms included in the device region. Next, a decision on the size of the electronic structure matrix, which is used to represent the bulk, must be made. This is equivalent to studying the extent that delocalization effects in the metal are important to describe correctly the bulk-conductor interaction. In the TB scheme, a smaller repeating unit implies more truncation of such long range effects. The TB calculation is implemented with the $\alpha$ and $\beta$ parameters [see Eq. (4)] obtained at the DFT level described below.

The choices that define the partition of the system are illustrated in Fig. 2. In the figure, $M$ is the number of metal atoms included with the device and $L$ is the total number of gold atoms included in the electronic structure calculation. $N$ is the number of bulk atoms included in the repeating TB unit. The size of the TB period defines the extent of truncation of long range electronic integrals included in the $\alpha$ and $\beta$ TB parameters. In this scenario, for example, all basis functions on atoms which are more than $N+M$ layers away from the edge (but still within $L$ ) are not included through the TB expansion. It is useful to note that this scheme does not vary the nature of the repeating unit but merely is a tool to determine the extent of electronic integral truncation within the coupling terms. A set of these three numbers defines the self energy model used in the transmission evaluation and will be referred to below by $L(M)-N$. Clearly for 
M (a) $\mathrm{N}=2$
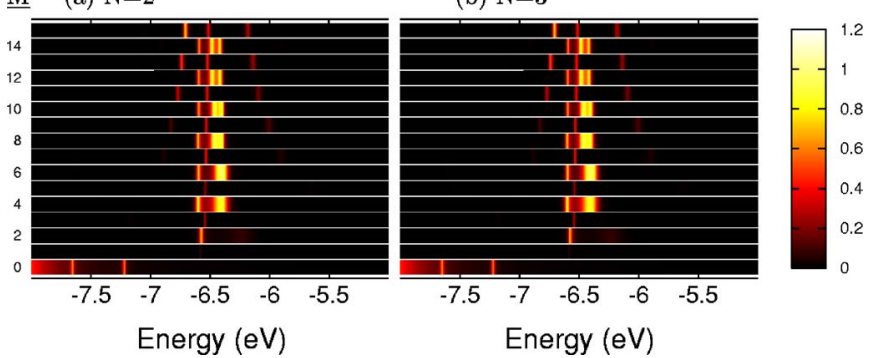

FIG. 3. Color-coded transmission plots of the nonpolar cumulene calculated with varying the space of included gold atoms with the device $(M)$. The bulk is represented by the WBL approximation. The sizes of the repeating period of the bulk are (a) $2(N=2)$ and (b) $3(N=3)$.

sufficiently large $L, M$, and $N$ values the model choice becomes converged as it does not introduce arbitrariness into the resulting calculated transmission. In the discussion below we study the radius of convergence of these choices for transmission calculations.

Electronic structure information is obtained for cluster models including the molecule and finite metal wires as described above. Twenty-four metal atoms are included on each side of the considered molecule, unless otherwise noted, at a density functional level of theory. The DFT calculation employs the B3LYP functional ${ }^{54,55}$ with a LANL2DZ ECP basis set $^{56}$ for the transition metal atoms and 6-31G for all other atoms. In all electronic structure calculations, the overall spin and charge of these systems, unless otherwise stated, is a neutral singlet. The electronic structure calculations were implemented using a prerelease version of QCHEM 3.0 (Ref. 57) and the transmission calculations were performed with a code developed in our group.

\section{RESULTS AND DISCUSSION}

Device $(M)$ convergence. We begin our benchmarking discussion by inspecting the convergence of the SE models with respect to $M$. This corresponds to the part of the electrode where screening effects and relaxation due to the adsorption are dominant. Therefore, $M$ corresponds to the number of gold atoms included in the contact region. Namely, we are considering the depth to which the surface adsorption causes a significant perturbation on the electronic structure of the electrode layers. First, we consider the plain nonpolar cumulene bonded between two gold atom chains. The calculated transmission functions are described by projection on a color contour, where the bright color region corresponds to $T=1$ and darker regions represent smaller transmission amplitudes. This allows us to follow clearly the convergence of a calculated series of transmission functions.

Figure 3 provides the projected calculated transmission functions for different $M$ values with the $L$ and $N$ values kept constant $[L=24$ and $N=2$ (left part) or $N=3$ (right)]. All transmission plots involve a single peak near the Fermi energy (FE) of the bulk electrode. At the WBL level of bulk representation there is no influence on increasing the bulk period size ( $N$ value). This is confirmed by comparing the two parts of the figure. However, the plots highlight a parity divergence, where the height of the peak converges differ-

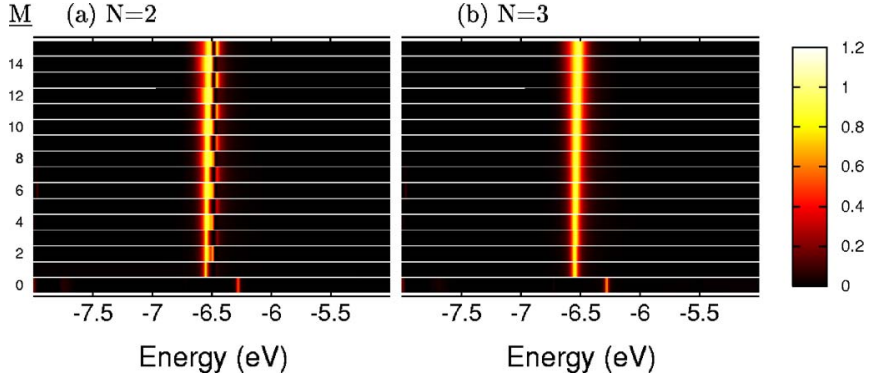

FIG. 4. Color-coded transmission plots of the nonpolar cumulene calculated with varying the space of included gold atoms with the device $(M)$. The bulk is represented by an explicit TB calculation. The sizes of the repeating period of the bulk are (a) $2(N=2)$ and (b) $3(N=3)$.

ently depending on whether $M$ is odd or even. Therefore, the use of SE models calculated with the WBL description for the bulk must ensue in arbitrary performance which depends on the $M$ value.

Next we consider the same sequence of transmission plots with, however, a full TB treatment of the bulk. Figure 4 describes the corresponding plots. With the TB bulk treatment, it is apparent that the odd-even parity can be lifted. However, while most of the disparity is eliminated already with $N=2$ plots (left side of the figure), almost complete elimination of the remaining divergence is demonstrated with an $N$ odd value ( $N=3$ in the right side). This small $M$ parity with even $N$ is removed only for a large enough $N$ value. Namely, with odd $N$ values a much faster convergence of the transmission plots is generated. This trend is demonstrated in Fig. 5, where the functionalized cumulene is considered. In the figure we follow the differences between devices defined with odd and even $M$ values (4 and 5) when coupled with an even or odd bulk $N$ value. In part (a) the transmission curves with even $N$ values are shown to reduce the parity only at $N=6$ or 8 , where the two curves with $N$ $=2$ are shown to differ substantially. In part (b) the use of an odd $N$ parameter leads to a faster convergence at $N=3$ or 5 . In (c) the convergence of the odd and even with sufficiently large $N$ cases is demonstrated. Next, we further consider the generality of the observations by calculating the $M$ sequence of the transmission plots with the more complex cumulene which involves the polarizing groups.

The $M$ dependence plots of the functionalized cumulene using the WBL and TB approximations for modeling the bulk are provided in Fig. 6 (WBL) and 7 (TB). The transmission plots in the figures are calculated with a small imaginary shift value (smear factor) added to the Hamiltonian of the device region when solving for the device GF as in Eq. (3). For the curves in part (b) (right side) a larger device shift factor is employed. This larger value further "smears" the electronic structure features of the MW and therefore highlights the main features of the molecular transmission pattern, where the dominant broadening effects due to the bulk are emphasized.

Indeed, the polarized cumulene transmission plots feature more structure as expected when compared to the plain cumulene results. However, the main observed transmission features confirm our discussion comparing the TB and WBL based approaches. Namely, the use of the TB model in cal- 


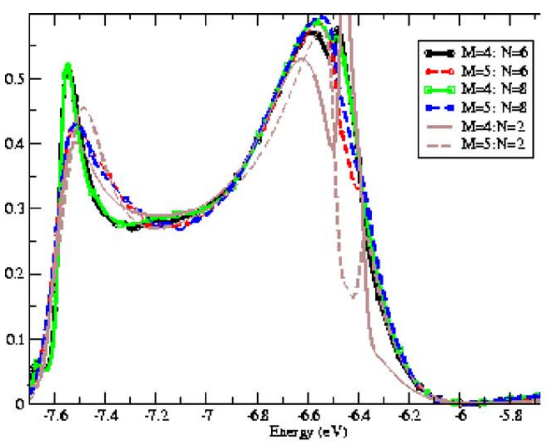

(a) Even bulk

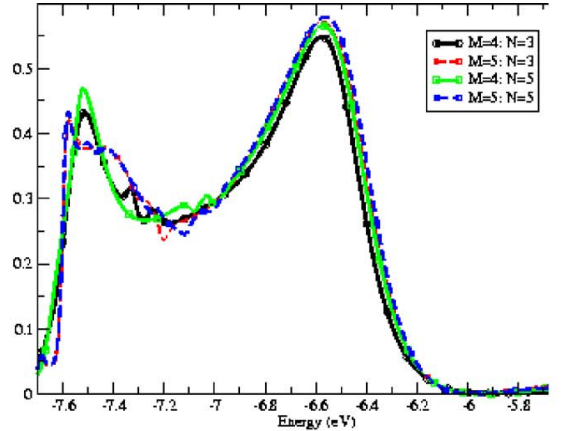

(b) Odd Bulk

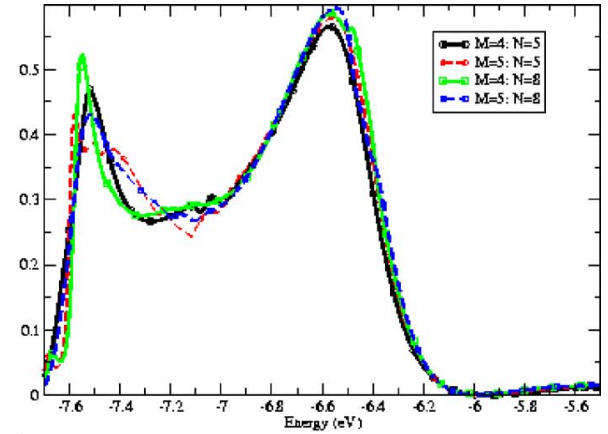

(c) Bulk Convergence

FIG. 5. Transmission plots of the polar cumulene. The disparity between using four or five gold atoms in the device is compared for different choices of the bulk parameter $(N)$. Transmission (a) for even $N$ value convergence at $N=8$ and (b) for odd $N$ value convergence at $N=5$. (c) Converged transmission for odd and even $N$ at $N=5$ and $N=8$.

culating the bulk's GF results in a converging model. Evidently the performance of the crude WBL approximation for describing the bulk (Fig. 6) in the calculation of transmission through the polarized cumulene is even worse than for the nonfunctionalized cumulene. The WBL plots clearly demonstrate a divergence that cannot be reduced by increasing the $N$ bulk parameter. This observation is confirmed also as outlined above after using a a larger shift factor. This factor is added to the device Hamiltonian in obtaining its GF. The same observations on the WBL divergence of the transmission function are maintained with the larger factor [see Figs. 6(a) and 6(b)]. The TB curves illustrated in Fig. 7, on the other hand, provide a convergent series.

Next we comment on the origin of the difference in the $M$ performance for the WBL and TB based models. In WBL, the device transmission pattern is determined by the parity of the last atom included in the device space. The coupling to the simplified bulk models in the WBL is unable to compensate for the device electronic structure differences between including an even or odd number of bulk atoms in the device subspace. This situation is rectified by using a more complex bulk GF generated by the explicit TB treatment of the bulk. Namely, the TB is capable of producing a consistent description by appropriately coupling to a variety of electronic states in the device region as evident from the $M$ series transmission plot. These trends persist in both singlet and triplet spin coupling schemes. This has been confirmed by performing a variety of specialized transmission function evalua-
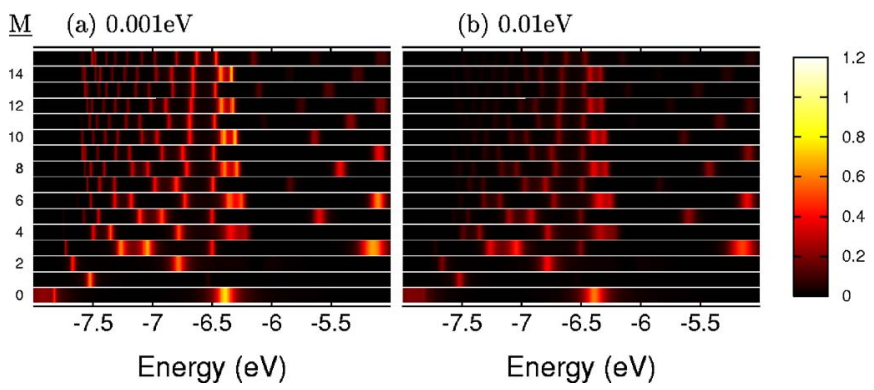

FIG. 6. Color-coded transmission plots of the polarized cumulene calculated with varying the space of included gold atoms with the device $(M)$. The bulk is represented by a WBL approximation with $(N=3)$. The shift factors used in calculating the device GF are (a) $0.001 \mathrm{eV}$ and (b) $0.01 \mathrm{eV}$. tions. We note, however, that TB still produces a small divergence with respect to the $M$ factor. The effect of this small divergence on calculated conductance or $I-V$ plots is, however, negligible. In addition, this can be further reduced by increasing the $N$ parameter. However, the $M$ convergence is slower for even values of $N$, while the performance of $M$ series with odd values of $N$ is shown to provide a faster converging set of plots.

Most of the remaining $M$ value related divergence observed at the TB level is in the form of minor changes in the transmission function at around $-7.6 \mathrm{eV}$, which are emphasized by the smaller shift factor [Fig. 7(a)]. The smaller shift factor allows expression of these features in the transmission plots. These originate from the electronic DOS of the gold atoms included with the device. The number of these localized peaks increases with the number of gold atoms included within the device. However, their overall contribution to conductance is minute since these are not broadened significantly and are well distant from the FE. Overall, it is important to note that both sets are shown to converge to similar transmission patterns. Therefore consistent conductivity plots correspond to these converged $M$ value series. To summarize this discussion, the crude description employing the WBL approximation for the bulk fails to provide consistent transmission with respect to the device parameter. This highlights the need for the more accurate TB description of the bulk. These observations are noted also for the calculated trans-
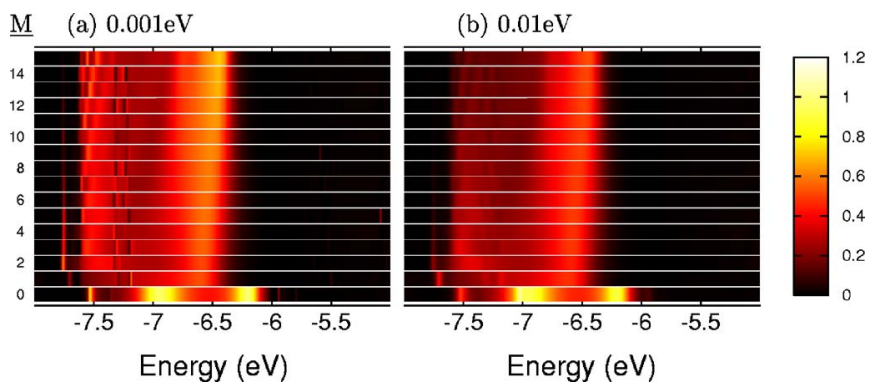

FIG. 7. Color-coded transmission plots of the polarized cumulene calculated with varying the space of included gold atoms with the device $(M)$. The bulk is represented by an explicit TB calculation with $(N=3)$. The shift factors used in calculating the device GF are (a) $0.001 \mathrm{eV}$ and (b) $0.01 \mathrm{eV}$. 


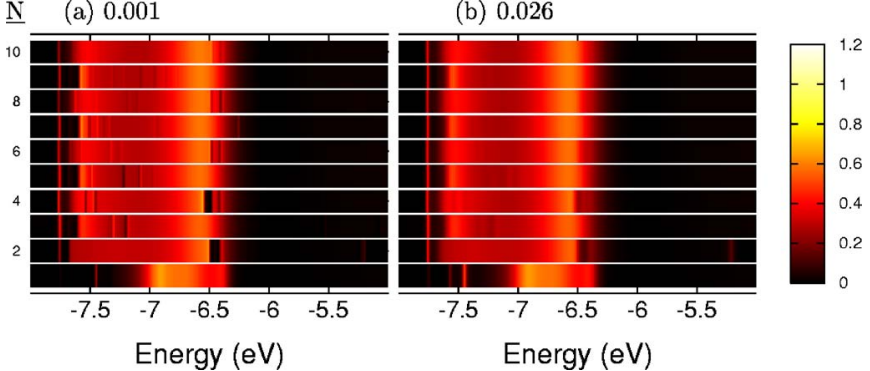

FIG. 8. Color-coded transmission plots of the polarized cumulene calculated with varying the size of the repeating unit for describing the bulk. The device space is set constant $(M=3)$. The shift factors used in calculating the bulk GF are (a) $0.001 \mathrm{eV}$ and (b) $0.026 \mathrm{eV}$.

mission of the other systems considered in this work. Next, we study the convergence of the bulk size parameter included in the TB model.

Bulk $(N)$ convergence. We now turn to study the convergence of the SE models with respect to the size of the space used to define the repeating unit in the bulk's TB calculation. A larger $N$ factor allows a more complete description of bulk electronic delocalization effects through the TB parameters, where electronic integrals over atomic orbital indices with larger inter-bulk-atom distances are included. This is the size of the subspace that is considered to be the repeating unit in the bulk periodic material. The transmission curves calculated with varying $N$ values and setting $M=3$ and $L=24$ are plotted in Fig. 8. The provided plots demonstrate an overall fast convergence with respect to this bulk unit size parameter. The two parts of the figures correspond to different shift factors which are used with the bulk's GF calculation.

We first focus on the plots provided on the left side of the figure, which employ the smaller bulk shift factor. Several features related to the bulk model are shown to converge slowly with the $N$ value. These are manifested as strongly localized deep wells and peaks of the overall transmission plot, which are eliminated when a large enough bulk is included. This demonstrates the effect of using a truncated bulk model for describing a metallic system. Only large enough models, which include enough of the bulk long range interactions, can better describe the delocalized nature of the metallic bulk. We note, however, that these discretization effects also have only a marginal effect on the integrated transmission (of course only when a sufficiently large bulk space is used).

These cluster effects in the bulk model can also be treated within the bulk GF calculation. With the larger smear factor the convergence of the curves is even faster with $N$ $=3$ or 4 as provided in the right part of the figure. These observations are also consistent for all the considered systems, including the $\mathrm{Al}$ bulk calculations, the various molecular orientations, and the polarized and plain cumulene systems. We next turn to study the effect of the cluster model size used in the electronic structure calculation on the evaluated transmission.

Electronic structure cluster model $(L)$ convergence. Next, we consider the effect of using a truncated cluster model for describing the interaction of the bulk with the transporting molecule. This is implemented by repeating the

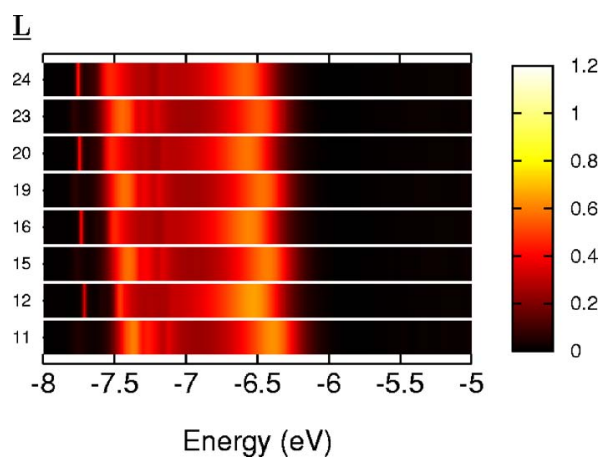

FIG. 9. Color-coded transmission plots of the polarized cumulene calculated with varying the number of gold atoms included in the model used in the electronic structure calculation $(L)$.

transmission calculation with varying numbers of bulk atoms included in the electronic structure calculation. This amounts to repeating the evaluation of the supermolecule electronic structure with a modified $L$ value. The transmission plots where $N$ and $M$ are each set to 3 and with the changing $L$ values are provided in Fig. 9.

In Fig. 9 different limits for the odd and even cases of the $L$ value are demonstrated. It is also apparent that fast convergence is exhibited for the even and odd cases separately. Not more than three atoms over the size defined by $N+M$ are needed to converge each of the odd or even series. The few atoms added beyond $N+M$ atoms reduce small edge effects. These edge effects are shown to only shift or increase moderately the transmission peaks. As noted above, however, the results indicate a disparity of the transmission between the odd and even $L$ values. This divergence originates from the open shell character of the gold atoms. This is indicated also by simply noting that the highest occupied molecular orbitals-lowest unoccupied molecular orbital (HOMO-LUMO) gap and energies depend on the parity of the number of gold atoms included in the electronic structure calculation. Therefore, an essentially different transporting material is modeled by merely choosing the number of gold atoms in the cluster model calculation. This stems from the different inter-atomic spin coupling schemes involved with varying the parity of the number of open shell metal atoms included in the model.

\section{CONCLUSIONS}

Transport between two metallic bulks through molecular systems can be viewed as a coherent process involving the whole system. In order to treat this at a (at least) well converged qualitative level a large enough cluster model representing the bulk must be included in the electronic structure description. This must include in the device the dominant terms describing the chemical changes induced by the adsorption of the molecule to the bulk surface. In this report we have studied the convergence of self energy models that represent the influence of the bulk on the molecular electronic levels and their effect on transport through these levels.

We have considered different model systems consisting of $\mathrm{Al}$ and $\mathrm{Au}$ electrodes and molecular cumulenes where polarizing groups have been added. The polarizing groups al- 
low consideration of richer transmission functions. We have also varied the considered orientation of the MW with respect to the electrode. The effect of choosing the regions within the cluster model used to represent the bulk and the device has been studied. This benchmarking study provides the underlying conditions for defining self-energy models that lead to a consistent calculated transmission function.

Arbitrary (nonconvergent) results emerge when chemically modified electrode atoms are not included within the device region. Furthermore, the different levels for representing the bulk through its approximated Green's function may also lead to arbitrary results even when working with sufficiently large device regions. This is demonstrated by comparing the transmission calculated with the bulk represented at a WBL approximation to a DFT based TB treatment. It is shown that most of the numerical divergence introduced by the crude WBL level can be corrected by using an appropriate explicit TB approach. We have also seen that the overall cluster model which is used to describe the bulk must be defined carefully as this may entail representing conducting materials with different electronic structure. This is related to the open shell nature of the gold atoms and also depends on whether the molecule is chemically bonded to the surface or only physisorbed. Additional studies for generalizing the recommendations for constructing converged self-energy models to more than one dimensional studies are required. We will also implement more sophisticated self-energy expressions, that allow more rigorous treatment of the strong interaction between bulk atoms and the conducting molecule. This will further enhance the convergence of the SE model with respect to the sizes of the different considered models.

\section{ACKNOWLEDGMENTS}

One of the authors (B.D.D.) acknowledges the University of Michigan for financial support. The authors also thank Shai Kendler in assisting them with the figure preparation.

${ }^{1}$ C. M. Fischer, M. Burghard, and S. Roth, Synth. Met. 71, 1975 (1995).

${ }^{2}$ M. A. Reed, C. Zhou, C. J. Muller, T. P. Burgin, and J. M. Tour, Science 278, 252 (1997).

${ }^{3}$ J. Chen, M. A. Reed, A. M. Rawlett, and J. M. Tour, Science 286, 1550 (1999).

${ }^{4}$ C. P. Collier, E. W. Wong, M. Belohradsky, F. M. Raymo, J. F. Stoddart, P. J. Kuekes, R. S. Williams, and J. R. Heath, Science 285, 391 (1999)

${ }^{5}$ C. P. Collier, G. Mattersteig, E. W. Wong, Y. Luo, K. Beverly, J. Sampaio, F. M. Raymo, J. F. Stoddart, and J. R. Heath, Science 289, 1172 (2000).

${ }^{6}$ T. Rueckes, K. Kim, E. Joselevich, G. Y. Tseng, C. L. Cheung, and C. M. Lieber, Science 289, 94 (2000).

${ }^{7}$ C. Joachim, J. K. Gimzewski, and A. Aviram, Nature (London) 408, 541 (2000).

${ }^{8}$ A. Nitzan, Annu. Rev. Phys. Chem. 52, 681 (2001).

${ }^{9}$ J. M. Tour, A. M. Rawlett, M. Kozaki et al., Chem.-Eur. J. 7, 5118 (2001).

${ }^{10}$ F. R. F. Fan, J. P. Yang, L. T. Cai, D. W. Price, S. M. Dirk, D. V.
Kosynkin, Y. X. Yao, A. M. Rawlett, J. M. Tour, and A. J. Bard, J. Am. Chem. Soc. 124, 5550 (2002).

${ }^{11}$ J. Park, A. N. Pasupathy, J. I. Goldsmith et al., Nature (London) 417, 722 (2002).

${ }^{12}$ W. J. Liang, M. Shores, M. P. Bockrath, J. R. Long, and H. Park, Nature (London) 417, 725 (2002).

${ }^{13}$ Y. Luo, C. P. Collier, J. O. Jeppesen et al., ChemPhysChem 3, 519 (2002).

${ }^{14}$ D. M. Adams, L. Brus, C. E. D. Chidsey et al., J. Phys. Chem. B 107, 6668 (2003).

${ }^{15}$ R. M. Metzger, Chem. Rev. (Washington, D.C.) 103, 3803 (2003).

${ }^{16}$ A. Nitzan and M. A. Ratner, Science 300, 1384 (2003).

${ }^{17}$ R. L. McCreery, Chem. Mater. 16, 4477 (2004).

${ }^{18}$ W. Tian, S. Datta, S. Hong, R. Reifenberger, J. I. Henderson, and C. P. Kubiak, J. Chem. Phys. 109, 2874 (1998).

${ }^{19}$ R. Landauer, Philos. Mag. 21, 863 (1970).

${ }^{20}$ P. F. Bagwell and T. P. Orlando, Phys. Rev. B 40, 1456 (1989).

${ }^{21}$ I. Imry and R. Landauer, Rev. Mod. Phys. 71, S306 (1999).

${ }^{22}$ M. P. Samanta, W. Tian, S. Datta, J. I. Henderson, and C. P. Kubiak, Phys. Rev. B 53, R7626 (1996).

${ }^{23}$ S. N. Yaliraki, A. E. Roitberg, C. Gonzalez, V. Mujica, and M. A. Ratner, J. Chem. Phys. 111, 6997 (1999).

${ }^{24}$ M. Di Ventra, S. T. Pantelides, and N. D. Lang, Phys. Rev. Lett. 84, 979 (2000).

${ }^{25}$ Y. Xue, S. Datta, and M. A. Ratner, Chem. Phys. 281, 151 (2002).

${ }^{26}$ K. Stokbro, J. Taylor, M. Brandbyge, J. L. Mozos, and P. Ordejn, Comput. Mater. Sci. 27, 151 (2002).

${ }^{27}$ N. D. Lang and P. Avouris, Phys. Rev. Lett. 84, 358 (2000).

${ }^{28}$ Y. Xue, S. Datta, and M. A. Ratner, J. Chem. Phys. 115, 4292 (2001).

${ }^{29}$ N. D. Lang and P. Avouris, Nano Lett. 3, 737 (2003).

${ }^{30}$ P. S. Damle, A. W. Ghosh, and S. Datta, Phys. Rev. B 64, 201403 (2001).

${ }^{31}$ J. Taylor, H. Guo, and J. Wang, Phys. Rev. B 63, 245407 (2001).

${ }^{32}$ P. Damle, A. W. Ghosh, F. Zahid, and S. Datta, Chem. Phys. 171-187, 225 (2002).

${ }^{33}$ Y. Xue and M. A. Ratner, Phys. Rev. B 68, 115406 (2003).

${ }^{34}$ M. Brandbyge, J. L. Mozos, P. Ordejon, J. Taylor, and K. Stokbro, Phys. Rev. B 65, 165401 (2002).

${ }^{35}$ M. Galperin and A. Nitzan, Ann. N.Y. Acad. Sci. 1006, 48 (2003).

${ }^{36}$ K. Hirose and M. Tsukada, Phys. Rev. B 51, 5278 (1995).

${ }^{37}$ N. Lang, Phys. Rev. B 52, 5335 (1995).

${ }^{38} \mathrm{~S}$. Datta, Electronic Transport in Mesoscopic Systems (Cambridge University Press, New York, 1995).

${ }^{39}$ S. H. Ke, H. U. Baranger, and W. Yang, Phys. Rev. B 70, 085410 (2004).

${ }^{40}$ G. C. Solomon, J. R. Reimers, and N. S. Hush, J. Chem. Phys. 122, 224502 (2005).

${ }^{41}$ B. Larade, J. Taylor, H. Mehrez, and H. Guo, Phys. Rev. B 64, 075420 (2001).

${ }^{42}$ P. Derosa and J. M. Seminario, J. Phys. Chem. B 105, 471 (2001).

${ }^{43}$ M. P. Lopez Sancho, J. M. L. Lopez Sancho, and J. Rubio, J. Phys. F: Met. Phys. 15, 851 (1985).

${ }^{44}$ M. B. Nardelli, Phys. Rev. B 60, 7828 (1999).

${ }^{45}$ T. Seideman and W. H. Miller, J. Chem. Phys. 97, 2499 (1992).

${ }^{46}$ O. Hod, R. Baer, and E. Rabani, J. Am. Chem. Soc. 127, 1648 (2005).

${ }^{47}$ O. Hod, E. Rabani, and R. Baer, Acc. Chem. Res. 39, 109 (2006).

${ }^{48}$ O. Hod, R. Baer, and E. Rabani, J. Phys. Chem. B 108, 14807 (2004).

${ }^{49}$ O. Hod, E. Rabani, and R. Baer, J. Chem. Phys. 123, 051103 (2005).

${ }^{50}$ B. Xu and N. Tao, Science 301, 1221 (2003).

${ }^{51}$ X. Xiao, B. Q. Xu, and N. Tao, Nano Lett. 4, 267 (2004).

${ }^{52}$ G. Yanson, A. I. Rubio Rollinger, H. E. van der Brom, N. Agrait, and J. M. van Ruitenbeek, Nature (London) 395, 783 (1998).

${ }^{53}$ H. Ohnishi, Y. Kondo, and Takayanagi, Nature (London) 395, 780 (1998).

${ }^{54}$ A. D. Becke, J. Chem. Phys. 98, 1372 (1993).

${ }^{55}$ A. D. Becke, J. Chem. Phys. 98, 5648 (1993).

${ }^{56}$ W. R. Wadt and J. P. Hay, J. Chem. Phys. 82, 299 (1985).

${ }^{57}$ Y. Shao, L. Fusti-Molnar, Y. Jung et al., Phys. Chem. Chem. Phys. 8, $3172(2006)$ 